\title{
Planning and Operational energy optimization solutions for Smart Buildings
}

\author{
David Sembroiz ${ }^{\mathrm{a}}$, Davide Careglio ${ }^{\mathrm{a}}$, Sergio Ricciardi ${ }^{\mathrm{a}}$, Ugo Fiore ${ }^{\mathrm{b}}$ \\ ${ }^{a}$ Department of Computer Architecture, Technical University of Catalonia - BarcelonaTech (UPC), Spain \\ ${ }^{b}$ Department of Management and Quantitative Studies, Parthenope University, Italy
}

\begin{abstract}
The incremented popularity of Internet of Things (IoT), thanks to improvements both in hardware and software of sensors over the last years, enables the possibility to monitor and gather any kind of data. Additionally, the arrangement of heterogeneous sensors, capable of perceiving information about their surroundings, into a rich Wireless Sensor Network (WSN), allows the appearance of complex systems in which resources are managed more efficiently. Smart cities, buildings, parkings, emergency services are appearing, where control over energy consumption and better sustainability are coupled with an improvement of the comfort of occupants. In this paper, we address the problem of energy optimization in smart buildings, considering both the planning and operational aspects. Specifically, the first aim is to propose an optimal deployment of the WSN inside a building. For this, we present a model able to identify the optimal locations for different types of sensors and gateways, by optimizing energy consumption while fulfilling connectivity, resource, protection, and clustering coverage constraints. Once the IoT system is deployed, we address the problem of how the building actually functions, according to the behaviour of the occupants. In particular, we propose a Building Management System (BMS) capable of efficiently and automatically manage the building elements using human behavioural models, thus lowering the overall building energy consumption whilst maintaining acceptable levels of comfort.
\end{abstract}

Keywords: Internet of Things, Smart buildings, optimization, energy, comfort, Wireless Sensor Network, ILP, simulation

\section{Introduction}

Internet of Things (IoT) is increasingly gaining popularity, due to technology improvements both in hardware and software, such as miniaturization, energy efficient sensors, lightweight communication protocols, which enable new possibilities for measuring, monitoring and interacting with the environment. IoT is commonly defined as a system of interconnected elements or things that are provided with unique identifiers and the ability to gather and transmit information about - and exercise control over - any kind of element without necessity of human intervention. The list of elements is wide and can range from devices, machines or objects to animals or people. Thereby, a thing can be a person with a heart-rate monitor, an animal with a tracking system, a vehicle with sensors to alert the driver when tire pressure is low.

The aforementioned examples make use of individual sensors to achieve their goal. However, IoT also enables the possibility to interconnect multiple sensors to create a Wireless Sensor Network (WSN), which serves as the basis for more complex systems such as smart cities, buildings, parkings, emergency services, etc. [15].

Sensors are small pieces of hardware with constrained resources in terms of transmission range and, most importantly, battery life. Optimization of WSN deployment is key to achieve a network capable of delivering all the data whilst utilizing the least possible amount of resources. This optimization may follow different directions. For instance, in a factory where sensors are placed in locations with hard or limited reachability (for example locations with critical temperature conditions or high-rise places) energy efficiency is key. On the other hand, in a more easyto-deploy environment where full availability is a strict requirement, sensors might consume more energy to enable multiple transmission routes in the event of an unexpected partial network failure.

Furthermore, optimization of resources in a complex system can be extended beyond the WSN itself. Sensors can gather information about the status of different elements of the environment, and create smarter action policies 
capable of utilizing available resources more efficiently while preserving the desired behaviour. For instance, in a smart building, by monitoring elements such as air conditioners, lights, computers, windows, doors, etc. it is possible to automatically configure the environment according to the human behaviour and efficiently manage energy consumption. However, such optimization may come with a cost, as there is usually a clear trade-off between energy efficiency and human comfort: the higher the energy savings, the lower the comfort.

In this work, we focus on these aspects and study the energy optimization of a smart building. First, we address the optimal deployment of a WSN inside a building. For this, we present a model able to identify the optimal locations for different types of sensors and gateways, by optimizing energy consumption while fulfilling connectivity, resource, protection, and clustering coverage constraints. Second, we address the problem of the actual functioning of the building according to the behaviour of its occupants. In particular, we aim at developing a Building Management System (BMS) capable of efficiently and automatically manage building elements using human behavioural models, and, thus, try to lower the overall building energy consumption whilst maintaining acceptable levels of comfort. This can be achieved, for instance, by avoiding wasteful scenarios such as leaving lights on when rooms are empty, or by managing heating, ventilation and air conditioning (HVAC) systems more efficiently in order to offer a thermal comfort adequate to the actual occupation of the building.

The rest of the paper is organized as follows. Section 2 reviews the published related work for the sensor and gateway optimization problem and, then, we focus on reviewing the work done in the field of smart building simulation and occupant comfort calculation. Section 3 initially states the WSN optimization problem and subsequently defines the formulation of such model. Likewise, Section 4 firstly describes the smart building resource management problem and closes with an explanation of the smart building system. Results in Section 5 initially present the optimized WSN obtained and the energy impact induced by adding protection to the network. Then, results are shown about energy consumption and occupant comfort for the smart building, comparing them with the current scenario to decide, in Section 6, whether the enhancement of a building with smart features is beneficial for occupants. Section 6 also outlines future research aimed at improving the smart building features with superior prediction techniques.

\section{Related Work}

The problem of optimizing a WSN can be tackled from many different perspectives, depending on the objectives to optimize and the requirements, such as, e.g., topological restrictions, battery constraints, QoS requirements, and levels of resilience and security. Regarding the optimization objectives, some problems are related to reducing the initial WSN deployment cost by minimizing the number of nodes to install, while others focus more on WSN performance, by trying to minimize the overall network latency, maximize network connectivity to increase resilience in the event of unexpected failures, or minimize network energy consumption (especially in scenarios with constrained devices) [2].

With the appearance of IoT, WSNs have gained popularity due to the necessity to monitor every physical device in a given environment. Such requirement has brought WSNs into a more heterogeneous perspective, since the characteristics of sensors may vary depending on the features being monitored.

Additionally, new scenarios are no longer restricted to floors or 2D locations. Instead, IoT enables the possibility to monitor 3D environments such as office buildings, hospitals, cities, etc. Even though our research can handle both 2D/3D scenarios due to the chosen modelling and transmission assumptions, a more accurate 3D model which also takes into account line of sight would as in [14] would be necessary.

The utilization of heterogeneous sensors enriches the optimization problem by requiring proper positioning of each type of sensor in a specific region, instead of only choosing the positions in which sensors must be placed. This problem can be seen as a clustering problem in which every cluster region must contain a predefined set of sensors. Authors in [3] present an initial approach to such problem, restricting the positioning of every type of sensor into a subsection of the overall layout. However, the authors do not consider the possibility to overlap clusters of different sizes and thus the possibility to reduce the number of required set of sensors.

In addition to sensor placement, beacon or gateway placement is also another important factor in WSN optimization. The position in which gateways are deployed is crucial for connecting the overall network. Authors in [19] present an ILP model capable of locating the optimal beacon positions given a set of already selected sensor locations. Similarly, given a set of sensors, it is possible to determine the best gateway locations depending on the objective to optimize [4, 7]. However, the lack of clustering and sensor differentiation makes the model incomplete for IoT. In [8], 
a gateway placement with QoS constraints is presented in which clusters are created around the deployed gateways, which differs from our definition of cluster. In [6], authors show a model capable of selecting, from a given Wireless Mesh Network, the nodes that should act as gateways, instead of placing additional specific nodes.

Many research papers are also focused in the optimization of building operational energy consumption by means of smart systems (e.g., [10, 20]). Additionally, some authors introduce the comfort of the occupants to check whether a trade-off between those two metrics exists [21, 17, 11, 16, 24]). These studies are the starting point of present work. For solving the operational problem of improving energy efficiency in a building while maintaining acceptable levels of comfort of the occupants, it is needed to quantify such comfort first. To this aim, several equations are presented in the literature [18, 5]. Authors in [21] propose the usage of an aggregated equation that weighs and combines several independent comfort values, such as thermal, light, air quality and acoustic comforts. This is utilized as the basis for our work, even though we require the insertion of additional comfort values. In the specific case of the temperature, the comfort is usually calculated following the ANSI/ASHRAE Standard 55 [1] Predicted Mean Vote (PMV) equation, that can also be extended to introduce air quality aspects [12, 17, 11]. Recommended light levels of the National Optical Astronomy Observatory are shown in [13]; such recommendations have been utilized in many works as the comfort values to aim for [24, 16].

Our work utilizes and extends the aforementioned literature by means of an initial planning model capable of calculating the optimal sensor and gateway positions for a given building layout. Even though this topic has been widely studied, our model introduces the concept of protection level to enable the possibility of equipping the network with backup paths in the event of a network failure. Furthermore, it also introduces overlapping clustering to determine not only the best sensor positions, but also the best type of sensor for each selected location. The concept of clustering already appears in many research articles regarding optimization problems, but we present the novel possibility to overlap positions into different clusters which is necessary for modelling efficient and complex IoT systems. Regarding the operational phase, we also extend the current comfort equations of the operational problem by inserting additional values, and, thus, show how comfort equations can be modelled and adjusted to fulfill different scenarios.

\section{WSN deployment optimization}

This section presents the placement optimization problem for sensors and gateways in a smart building. Firstly, in Section 3.1, we formulate the problem, identifying the constraints we are considering in this scenario and presenting the objective function, which entails the minimization of energy consumption. Furthermore, in Section 3.2, we progressively introduce all parameters and design assumptions considered in this specific placement problem and, finally, we present the model using a Mixed Integer Programming (MIP) formulation.

\subsection{Problem statement}

Given a building layout with known possible physical positions for placing either sensors or gateways, the problem resides in selecting the optimal locations for their deployment, minimizing the energy consumption of the whole system while fulfilling connectivity, resource, protection, and clustering coverage constraints. As the transmission range of a sensor is limited to few meters, the connectivity constraint ensures that a sensor is able to reach at least one gateway. The resource constraint refers to the maximum bandwidth of the gateway, i.e., the number of concurrent transmissions a gateway can receive from sensors. The protection constraint is related to unexpected gateway failures, meaning that if a gateway fails, the sensors transmitting to it need to be able to reach another gateway nearby. Finally, a cluster is defined as an area of the building where sensors from a given set of types are compulsorily required. It is worth mentioning that the size of clusters and the sensors required inside each cluster vary depending on the zone under representation. For instance, just a single alarm is placed in each floor of the building, so then, in this case, the cluster represents a floor; temperature, presence, and luminosity sensors are placed in each room, which form room clusters; a single humidity sensor is installed every four rooms to create clusters of room groups, etc. Besides, the possible sensor positions are not exclusively attached to a single cluster; instead, they can be shared in order to create a cluster hierarchy, which specifies different cluster sizes that cover different building zones with different sensor types.

Given such constraints, the objective of the placement optimization problem is to decide which locations to select in order to minimize energy consumption, given by the energy consumed by the sensors to communicate with the gateway plus the energy required by the gateways to collect data and forward them to the building manager. 


\subsection{Model formulation}

This section presents the WSN model that determines sensor and gateway positions inside a building aiming at minimizing the overall energy consumed.

We represent a building scenario as a set of potential sensor positions $S$ and a set of potential gateway positions $G$, each of which may correspond to a $2 \mathrm{D}$ or $3 \mathrm{D}$ point. Once the coordinates are marked, the variable dist of size $|S| \times|G|$ defines, for each element $d i s t_{i j}$, the Euclidean distance between positions $S_{i}$ and $G_{j}$. Figure 1 shows an example of a building layout where blue dots represent possible sensor positions and red dots indicate possible gateway positions.

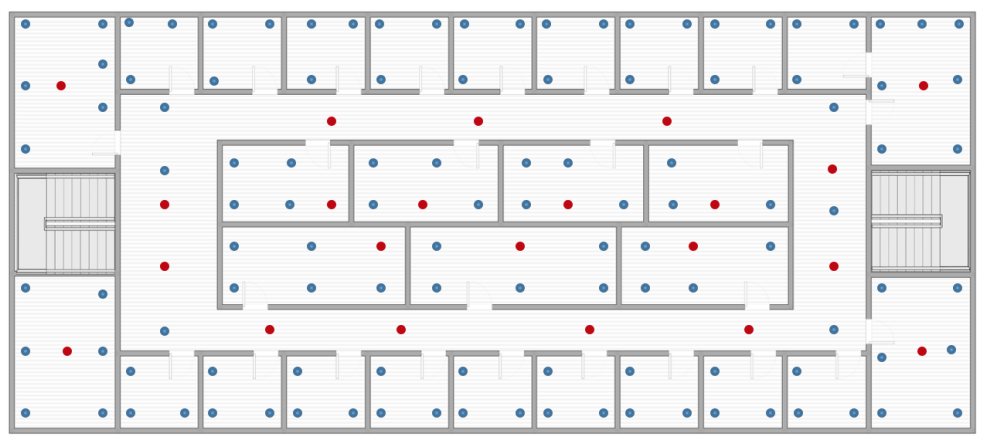

Figure 1: Layout of the building with the sensor and gateway available positions defined.

The locations where sensors and gateway should be initially placed depend on the energy consumption model. The calculation of the sensors' energy consumption is directly related to the distance between sensors and their assigned gateway. We employ Mica2 motes [23] due to their flexibility to alter their transmission range in order to efficiently manage energy consumption, as shown in Table 1 . Specifically, transmissionCost $t_{i}$ stores the energy needed for sending data using power level $i$. To simplify further calculations, it is assumed that the energy of each level of the table corresponds to the transmission of a single data packet.

\begin{tabular}{ccc|ccc}
\hline$l$ & $E_{t x}(l)$ & $R(l)$ & $l$ & $E_{t x}(l)$ & $R(l)$ \\
\cline { 1 - 3 } $1\left(l_{\min }\right)$ & 671.88 & 19.3 & 14 & 843.75 & 41.19 \\
2 & 687.50 & 20.46 & 15 & 867.19 & 43.67 \\
3 & 703.13 & 21.69 & 16 & 1078.13 & 46.29 \\
4 & 705.73 & 22.69 & 17 & 1132.81 & 49.07 \\
5 & 710.94 & 24.38 & 18 & 1135.42 & 52.01 \\
6 & 723.96 & 25.84 & 19 & 1179.69 & 55.13 \\
7 & 726.56 & 27.39 & 20 & 1234.38 & 58.44 \\
8 & 742.19 & 29.03 & 21 & 1312.50 & 61.95 \\
9 & 757.81 & 30.78 & 22 & 1343.75 & 65.67 \\
10 & 773.44 & 32.62 & 23 & 1445.31 & 69.61 \\
11 & 789.06 & 34.58 & 24 & 1500.01 & 73.79 \\
12 & 812.50 & 36.66 & 25 & 1664.06 & 78.22 \\
13 & 828.13 & 38.86 & $26\left(l_{\max }\right)$ & 1984.38 & 82.92 \\
\hline
\end{tabular}

Table 1: Transmission energy consumption $\left(E_{t x}(l)\right.$ in $n J /$ bit $)$ and transmission range $\left(R_{(} l\right)$ in $\left.m\right)$ at each power level $(l)$ for the Mica2 motes as a function of power level [23]. Energy dissipation for reception of data is constant $\left(E_{r x}=922 n J / b i t\right)$.

For the energy consumption of the gateway, we consider the parameter $\operatorname{Cost} G_{j}$. This cost is assigned to each gateway inside the range [400, 1000], utilizing the same consumption units as the sensor transmissions.

Another important aspect that should be taken into account is the variety of sensors to deploy. IoT appeared thanks to improvements in sensor technology, which opened the possibility to gather any kind of data, e.g., temperature, humidity, luminosity, air quality, and fire alarm . Our model handles $T$ distinct types of sensors, each of which is responsible for gathering different environmental data. Even though their characteristics may vary, in the interests of simplicity we consider that transmission range and energy consumption are equal for all sensors and correspond to the values shown in the aforementioned Table 1 . For transmission, the maximum reachable range is limited to $R\left(l_{\max }\right)$. In 
the final model, the adjacency matrix InRange is defined:

$$
\text { InRange }_{i j k}= \begin{cases}1 & \text { if } i \in S, j \in G, k \in T, \operatorname{dist}(i, j) \leq R\left(l_{\text {max }}\right) \\ 0 & \text { otherwise }\end{cases}
$$

This adjacency matrix allows to readily find the possible connections that can be established between a sensor of type $k$ placed in position $S_{i}$ with a gateway placed in position $G_{j}$.

A novelty introduced in our WSN modelling is the utilization of clusters. Clusters are usually defined as a group of the same or similar elements gathered closely. Here, a cluster is defined as a set of sensor positions in which a group of sensor types needs to be exhaustively represented. This addition allows the creation of flexible solutions. The model considers $N_{c}$ clusters, and the variable clusterPositions stores, for each sensor position $i$, a list of the cluster identifiers $j$ to which position $i$ belongs. This definition enables the possibility to define regions that belong to more than one cluster, in order to describe more complex scenarios.

Figure 2 shows an example of cluster definition. As it can be seen, the model permits the definition of small clusters inside bigger ones. In this particular example, the entire floor belongs to the blue cluster, accommodated for sensor types that only need to be placed once per floor, such as a fire alarm. The green cluster consists of all rooms facing the top side of the building. Sensors placed in that area will gather information that does not significantly vary from one room to the contiguous ones, like air quality, humidity and gases detection. The red cluster represents a single room, where sensors like temperature, presence, luminosity, smart plugs, etc. have to be installed.

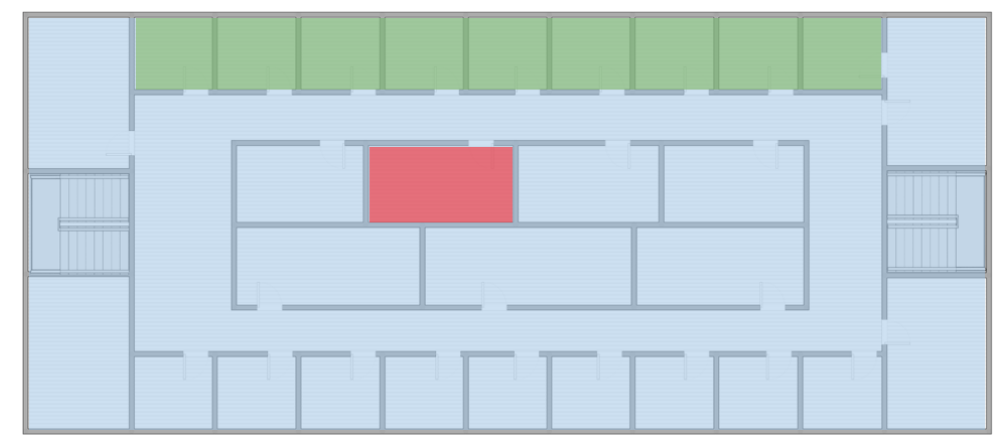

Figure 2: Example of three clusters of different size defined in a building floor.

Since the number of positions inside each cluster may vary, the final model uses an auxiliary adjacency matrix $c l u$, whose entry $c l u_{i j}$ determines whether position $S_{i}$ is inside the cluster with $i d=j$.

$$
\text { clu }_{i j}= \begin{cases}1 & \text { if } i \in S, 1 \leq j \leq N_{c}, j \in \text { clusterPositions }_{i} \\ 0 & \text { otherwise }\end{cases}
$$

In order to handle heterogeneous scenarios, another variable is needed to indicate the set of sensor types that each cluster must contain. To this aim, the variable clusterTypes is defined, and stores, for each cluster $i$, a list of sensor types $k$ that must be present. As before, since the number of sensor types needed per cluster may vary, an auxiliary adjacency matrix is used for the final model:

$$
\text { cluTypes }_{i j}= \begin{cases}1 & \text { if } 1 \leq i \leq N_{c}, j \in T, j \in \text { clusterTypes }_{i} \\ 0 & \text { otherwise }\end{cases}
$$

A final valid deployment is represented by a set of sensor locations $P S=\left\{1, \ldots, N_{s}\right\}$ and a set of gateway locations $P G=\left\{1, \ldots, N_{g}\right\}$. To this aim, the following set of boolean decision variables is defined to describe the selected 
gateway and sensor positions with their corresponding type, as well as the link between them.

$$
\begin{gathered}
x_{i j}= \begin{cases}1 & \text { if a sensor of type } j \text { is installed at position } S_{i} \\
0 & \text { otherwise }\end{cases} \\
y_{i}= \begin{cases}1 & \text { if a gateway is installed at position } G_{i} \\
0 & \text { otherwise }\end{cases} \\
a_{i j k}= \begin{cases}1 & \text { if sensor } i \text { of type } k \text { is attached to gateway } j \\
0 & \text { otherwise }\end{cases}
\end{gathered}
$$

Equation 4 shows the boolean variable that indicates whether position $S_{i}$ contains a sensor of type $j$. Similarly, Eq. 5 defines the analogous variable for the gateways; a vector suffices in this case since there is only one type of gateway. The last variable is presented in Eq. 6, and indicates the wireless link connections between sensors and gateways. Specifically, it determines whether a sensor of type $k$ in position $S_{i}$ is attached to a gateway positioned in $G_{j}$.

With all the decision variables defined, it is possible to clearly state the cost of deploying a sensor in terms of its associated gateway and the distance between them. To do so, we firstly calculate the index used for acquiring the proper transmission level as shown in Table 1 For the sake of simplicity, we assume that distances are rounded to the next unit.

$$
\text { index }=\left\lceil a_{i j k} * \operatorname{dist}(i, j)\right\rceil
$$

As it can be seen in Eq.7, index calculation is controlled by the decision variable $a_{i j k}$, in order to only count the transmissions in which sensor $i$ of type $k$ is attached to gateway $j$. It is worth mentioning that the index is always inside the range $\left[0,\left[R\left(l_{\max }\right)\right]\right]$. For index $=0$, nothing is found in Table 1 . However, this value is only obtained when $a_{i k j}=0$. Thus, it is safe to define transmissionCost $[0]=0$. Once the index is defined, transmissionCost [index $]$ returns the energy consumed for such transmission.

With all the required parameters and the variables defined, the proposed Mixed Integer Programming (MIP) model for solving the problem is as follows:

$$
\min \sum_{i \in S} \sum_{k \in T} x_{i k} * \sum_{j \in G} \text { transmissionCost }\left[\left\lceil a_{i j k} * \operatorname{dist}(i, j)\right\rceil\right]+\sum_{j \in G} \operatorname{Cost} G_{j} * y_{j}
$$

s. t.

$$
\begin{gathered}
2 * a_{i j k} \leq x_{i k}+y_{j} \quad \forall i \in S, j \in G, k \in T \\
\sum_{j \in T} x_{i j} \leq 1 \forall i \in S \\
a_{i j k} \leq \text { InRange }_{i j k} \quad \forall i \in S, j \in G, k \in T \\
\sum_{j \in G} a_{i j k} \geq x_{i k} * \text { protectionLevel }_{i} \forall i \in S, k \in T \\
\sum_{i \in S} \sum_{k \in T} a_{i j k} \leq \text { GatewayBw }_{k} \forall j \in G \\
\sum_{k \in S} \text { clu }_{k i} * x_{k j} \geq \text { cluTypes }_{i j} \quad \forall i \in C, j \in T
\end{gathered}
$$

The objective function $(8)$ minimizes the whole installation cost by minimizing the consumed energy for every sensor-to-gateway single packet transmission, as previously indicated, and the cost of deploying each gateway. The constraint 9 ensures that, if sensor position $S_{i}$ is associated with gateway location $G_{j}$, then, a sensor must exist at 
position $S_{i}$ and a gateway must be placed at position $G_{j}$. The constraint 10 ensures that each position $S_{i}$ holds one and only one type of sensor, since the same physical position cannot be used by two separate elements. The range constraint (11) checks that all sensor-to-gateway links created are in the transmission range of the sensor.

The protection level is defined to introduce resilience in the network. This level indicates the number of gateways within the range of which each sensor must be, in such a way that, if a gateway fails, the sensor can be connected to an alternative one. To do so, constraint (12) forces a sensor of type $k$ at position $S_{i}$ to be connected to at least protectionLevel gateways. The bandwidth constraint (13) ensures that all gateways handle no more than their maximum bandwidth. For the sake of simplicity, we define the maximum bandwidth of a gateway as the maximum number of sensors connected to it. In case a given protection level is applied, the backup connections are also considered since they need to be available in the event of a gateway failure. Finally, the clustering constraint (14) forces each cluster to contain all sensor types required by the problem definition.

\section{Smart building resource management}

In the previous section, we solve the planning problem determining the optimal placement of sensors and gateways. Upon such an optimal topology, we now focus on the operational phase. In particular, we propose a BMS capable of automatically modify its state in order to adapt to the environmental conditions and the behaviour of occupants. Firstly, in Section 4.1, we introduce the scenario and formulate the problem and its objective. Then, in Section 4.2, we describe the models designed to mimic the environmental conditions, such as temperature, the different possible actions of the occupants during a normal working day, and the transition states of the building actuators. Finally, Section 4.3 defines the two metrics considered of interest to evaluate the performance of the proposed resource manager.

\subsection{Problem statement}

In the operational phase, we aim at developing a BMS capable of efficiently and automatically manage building elements and, thus, try to lower the overall building energy consumption whilst maintaining acceptable levels of comfort for occupants.

Indeed, besides energy consumption, another important metric to take into account in the operational phase of building automation is the comfort of occupants. In other terms, the environmental conditions in the building should be comfortable and pleasant for all occupants, matching as much as possible their desires. It is clear that pursuing both maximum comfort and minimum energy consumption may lead to contradictory actuations. For this very reason, we take into account both metrics in the design of our BMS and its decision-making process. It is worth mentioning that we are not focussing on any specific architecture or protocol implementation. Examples of architectures that can be adopted to avoid scalability issues can be found in [20, 22].

The proposed BMS receives two different inputs: i) the environmental conditions such as temperature, luminosity, air quality, etc. and ii) the movements and behaviours of the occupants of the building. With the latter, the BMS sets up and maintains occupants behavioural models. These models contain a set of actions that occupants are likely to perform during the day. For instance, one action included in a model could be that a specific occupant is usually entering in a given room between 7.30 and 8.30 am with a $90 \%$ probability. Movements and actions of the occupants can be tracked using both indoor and outdoor location services like in [9]. In any case, the construction of these models is out of the scope of this paper and will be addressed in future works. On the contrary, we consider that these models are already available and are inputs of our solution.

Using these models, the BMS monitors the status of the different rooms and, according to the expected movements of occupants, operates the actuators in order to minimize the energy consumption while maintaining acceptable levels of comfort. In order to provide to the system the time to take such decisions, we introduce a prediction threshold in Section 4.2.3, so that the BMS can act in advance with respect to actual occupant actions. For instance, the BMS can turn the HVAC on in a given room so that the desired temperature is reached just before the expected arrival of the occupant. Or, if the occupants of a room are expected to go for lunch at 12.30 am for an hour, the BMS automatically turns off the lights in the room, places smart plugs in sleep mode, reduces the HVAC use, etc. just after the expected action is performed, without the need of human intervention. 


\subsection{System definition}

This section presents the different models developed for environmental conditions, occupant movements and actions, and actuators. Since many features depend on the type of the building (residential or commercial), this section also makes reference to the specific building used for the performance evaluation in Section 5 . Specifically, we consider an office building where occupants can be employees sharing rooms, area directors with their own offices, and external personnel like visitors, building caretakers, and janitors. Clusters of three different levels are considered: room, zone (a given set of rooms), and floor. Regarding the type of sensors that can be placed in a room, we consider (i) a single sensor able to gauge temperature and luminosity and to detect whether the room is empty and (ii) a sensor that detects the status of a computer. In zone clusters, humidity and gas detection sensors can be installed. Only one alarm sensor is placed in the floor cluster. Concerning the actuators, each room is supposed to contain HVAC, smart plugs, and actuators for windows and doors.

\subsubsection{Environment}

Environmental conditions have an important impact on the energy consumption of a building. In this work, we consider temperature, humidity, and luminosity. A model is provided for each of them. Table 2 shows the average, minimum and maximum values considered for these three parameters, empirically collected on a winter day in Barcelona. The difference between these values and the desired conditions by building occupants mandates the use of indoor HVAC and lights.

\begin{tabular}{l|ccc} 
Parameter & Average & Min & Max \\
\hline Temperature $\left({ }^{\circ} \mathrm{C}\right)$ & 10,8 & 6 & 16 \\
Humidity $(\%)$ & 41,87 & 29 & 56 \\
Luminosity (lx) & 505 & 20 & 1500 \\
\hline
\end{tabular}

Table 2: Average, minimum and maximum environmental condition values.

As it can be observed, temperature always lies below the desired level of $21^{\circ} \mathrm{C}$. During the morning, environmental temperature increases from its lowest value, and reaches the maximum at midday. During the evening, temperature starts to decrease and arrives to its minimum, again, at night. Similarly, luminosity starts to increase as the sun rises, and starts to decrease at evening until it reaches its lowest value at night. Humidity, however, follows the opposite tendency. During morning and night it stays high, whilst in midday it reaches its lowest values.

\subsubsection{Occupants}

The occupants of a building are an important external agent that needs careful modeling. Occupants are categorized with different profiles, each of which contains a set of actions that can be performed. Every action is associated to the probability of its occurrence, specified hourly across a full day. That is, a user profile $U$ contains the set of actions $a_{1}, a_{2}, a_{3}, \ldots, a_{n}$, where each action $a_{i}$ is represented as a set $a_{i}=\left\{p_{1}, p_{2}, \ldots, p_{24}\right\}$ of 24 values $0 \leq p_{i} \leq 1$, one for each hour.

The set of possible actions strongly depends on the type and use of the building. As we consider an office building, we define the set of occupant actions during a working day as \{enter, move, meeting, lunch, exit $\}$. Figure 3 shows the valid location transitions associated to these actions.

As it can be seen, the first action always needs to be enter. Once inside the building, any occupant can choose to move to a specific room, go for lunch or participate in a meeting. To exit the building, it is needed for the occupant to either be in a room or in a meeting. Exiting the building is also a requirement in order to terminate the simulation, as the building has to be empty at the end of the day.

The set of actions inside the building and their probability during a day depend on the role of the occupant. As remarked in the previous section, we consider three different roles: employees, area directors, and external personnel. A different user profile is assigned to each role. For instance, Figure 4 shows an example of the distributions of the area directors' actions during a working day.

It is worth mentioning that, currently, these occupants behavioural models have not been constructed using real data. This problem has been left for future works. On the contrary, in this paper, we are interested in testing whether 


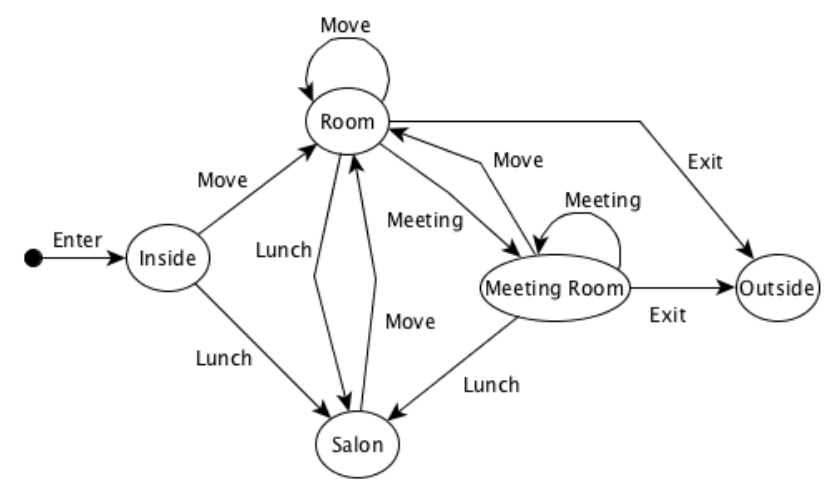

Figure 3: Location transition graph based on the current defined actions.

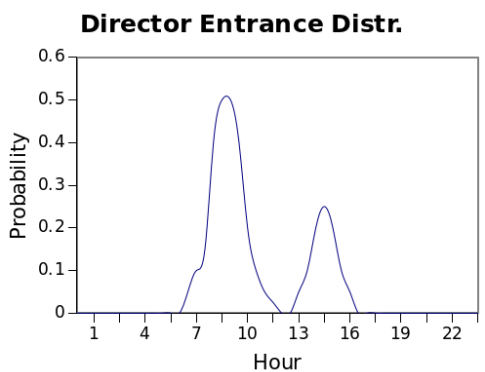

(a) Director probability distribution for the enter action.

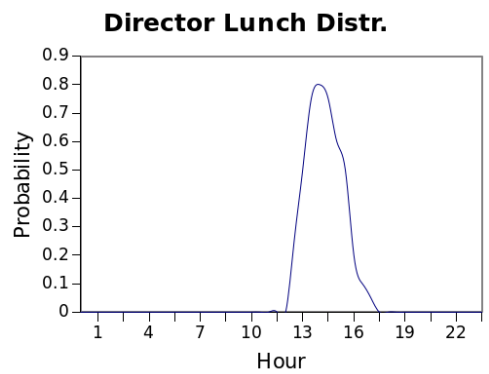

(c) Director probability distribution for the lunch action.

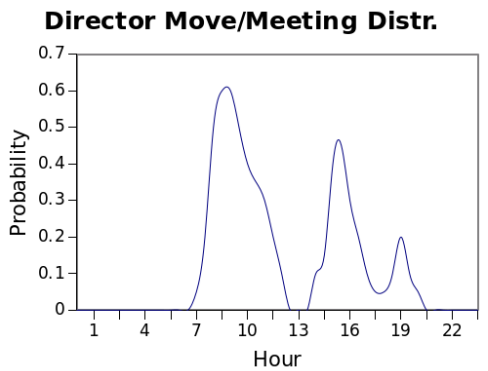

(b) Director probability distribution for the move and meeting actions.

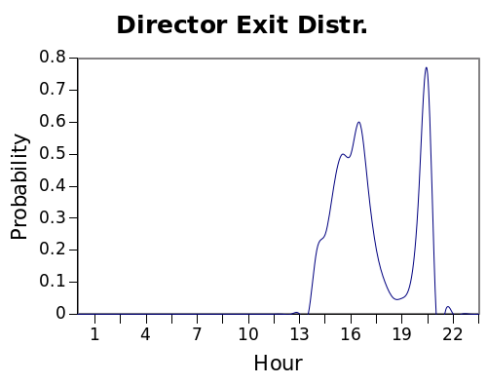

(d) Director probability distribution for the exit action.

Figure 4: Director user profile probability distributions.

taking smarter and automatic decisions in the BMS using these models effectively provides an improvement both in energy consumption and occupants' comfort.

\subsubsection{Actuation modes}

Our solution is able to launch automatic actuations for the different elements of the building in order to change their state, without the need of human intervention.

Figure 5 shows the state transition graphs for the different elements of the building, namely the HVAC, the lights and the smart plugs connected to the computers. Computers and HVAC can either be in off, suspended or on state while lights can be in off or on state. Each arrow is labeled with the combination of conditions required for executing the corresponding state transition. Specifically, we define the following conditions: 


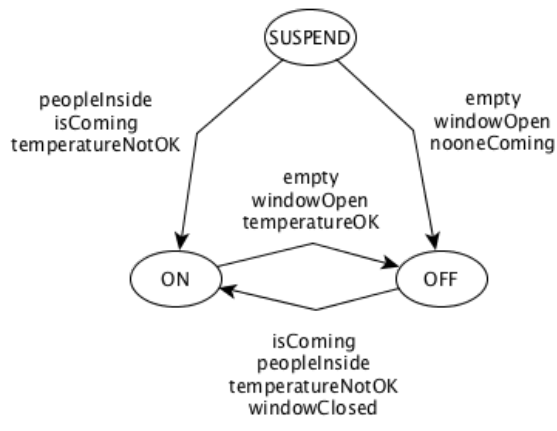

(a) Smart HVAC state transition graph.

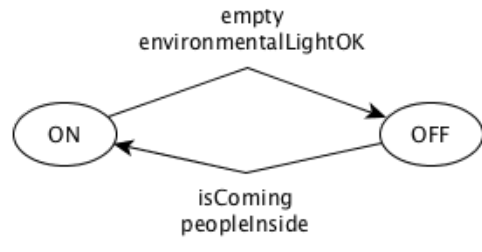

(b) Smart light state transition graph.

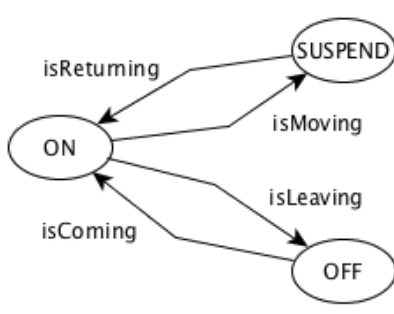

(c) Smart computer state transition graph.

Figure 5: State transition graphs for the monitored elements.

- peopleInside or empty, to detect whether there are people inside a room or it is empty;

- WindowOpen or WindowClosed, to detect if a window is either open or closed;

- TemperatureOK or TemperatureNotOK, to detect if the temperature is the one desired by occupants or not;

- environmemnatLightOK, to detect if the luminosity of the environment is enough and no artificial light is needed;

- isComing or isLeaving, to predict that someone is going to either enter in a room for the first time in the day or leave until the next day;

- isMoving or isReturning, to predict that someone is either moving outside or returning to a room from a meeting or lunch.

The last two items depend on the actions of the occupants. With the models defined in the previous section, the BMS is able to predict such actions. To give time to the BMS to react and prepare the building for the occupants, we define a prediction threshold. This threshold allows the BMS to act in advance with respect to the expected future actions, but only up to a certain interval. The value of this threshold clearly impacts both comfort and energy consumption; for this very reason, we perform an evaluation of such impacts in Section 5 in order to find the best trade-off.

Therefore, if, for instance, an occupant is going for lunch (isMoving), his computer is suspended; and it is brought back to on if the occupant is expected to return (isReturning) soon. We consider that the transition between the states of a computer requires 5 minutes. Similarly, if, for instance, a room is empty, its lights are switched off; but, if an occupant is expected to arrive (isComing) and the environmental luminosity is not OK, lights are turned on. In this case, the state transition is instantaneous.

The behaviour of the HVAC is slightly different, as the temperature cannot be adjusted instantaneously. To take this latency into account, we consider three parameters, namely the desired, the environmental, and the current temperature. The former is the temperature desired by the occupants of a room. When the HVAC of a room is on, the temperature gradually reaches the mean of the temperatures desired by the people inside; conversely, when the HVAC is either suspended or off, the temperature tends to the environmental one. We use Eq. (15) to update the temperature of a room. Every simulation step (10 seconds), a fraction of the absolute difference between the current and the desired temperature is either subtracted or added to the current temperature. The $F$ factor varies depending on the state of the HVAC. If the HVAC is on, the temperature varies at a $F=1 \%$ rate until the desired temperature is reached. If it is in either suspended or off state, the temperature changes at a $F=0.25 \%$ or $F=0.5 \%$ rate, respectively, until the environmental temperature is reached.

$$
T_{\text {new }}=T_{\text {current }} \pm\left|T_{\text {desired/environmental }}-T_{\text {current }}\right| * F
$$




\subsection{Metrics}

The two main metrics considered in our work are the overall building energy consumption and the comfort of the occupants. Following sections aim at defining such metrics in more detail.

\subsubsection{Energy consumption}

We consider that the building energy consumption depends on the HVAC systems, lights and computers. The energy consumed by the WSN may also be taken into account. The WSN is, however, considered fixed and immutable and therefore its consumption is constant and has been neglected in this part. Table 3 shows the power consumed by the three elements depending on their states. Thus, the overall energy consumption is calculated according to the state of these elements and their power consumption during the entire simulation time (1 day).

\begin{tabular}{l|ccc} 
Device & OFF & Suspended & ON \\
\hline HVAC & 0 & 800 & 1500 \\
Lights & 0 & - & 200 \\
Computer & 0 & 50 & 350 \\
\hline
\end{tabular}

Table 3: Energy (in kWh) consumed by the monitored devices in the different possible states.

\subsubsection{Comfort}

The definition of comfort has been widely studied in the literature and many different formulations have been proposed, as commented in Section 2. Many authors agree to consider thermal comfort as the most important parameter. However, since we are pursuing the automation of some elements in a smart building according to the expected actions of the occupants, we also include the adequacy of this automation in our formulation. Therefore, in our work, the definition of comfort aggregates several values with their proper weighting. In particular, thermal, light and device readiness comfort are considered.

The temperature comfort makes reference to the absolute difference between the desired temperature and the current one. The desired temperature is set to $21{ }^{\circ} \mathrm{C}$ and the system aims for delivering such condition once a person is expected to enter any room. In the event of a room shared by many people, the parameters are calculated as the mean of the desired values of the different occupants, in order to deliver a reasonable comfort level to all of them. Figure 6a shows the model used for assessing thermal comfort. It is worth noticing that when the desired and the current values widely differ $\left(4-6{ }^{\circ} \mathrm{C}\right)$, comfort is very low (lower than $20 \%$ ). However, small differences (less than or about $1{ }^{\circ} \mathrm{C}$ ) are better tolerated, with comfort close to $100 \%$. Reaching the maximum comfort, thus, may require substantial additional energy consumption and might be unnecessary.

Similarly, light comfort stands for the absolute difference between the desired and current room luminosity. Figure 6b shows the utilized luminosity model. The desired luminosity is set to 600 lux and, again, the system aims for delivering such value once a person is expected to enter any room. As for the case of the temperature, the luminosity model indicates a comfort close to $100 \%$ if the difference between the desired and the current value is small (less than or about 200 lux) and becomes very low (comfort close to 0 ) if the difference exceeds 400 lux.

Finally, the device readiness comfort is related to the prompt availability of the users' devices inside a room. For instance, computers must be fully operational when an occupant enters a room, instead of being off and needing to be turned on manually. For this case, no figure is shown since device readiness comfort follows a boolean tendency: when the computer is switched on and ready for use, the comfort is maximum, whereas it is 0 in all other cases (off, suspended or transitioning from one state to another).

The overall occupants' comfort formulation is provided in Eq. 16. The weights used for the thermal and luminosity comforts are similar to those proposed in [18]. However, we have added the device readiness comfort, assigning a weight of $5 \%$ to it in order to preserve the importance of both temperature and luminosity.

$$
C=0.57 * C_{\text {luminosity }}+0.38 * C_{\text {thermal }}+0.05 * C_{\text {device }}
$$




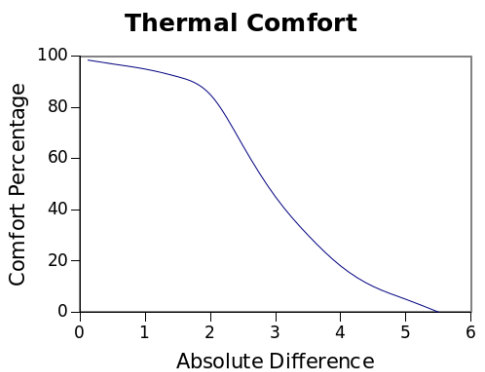

(a) Thermal comfort percentage depending on absolute temperature difference.

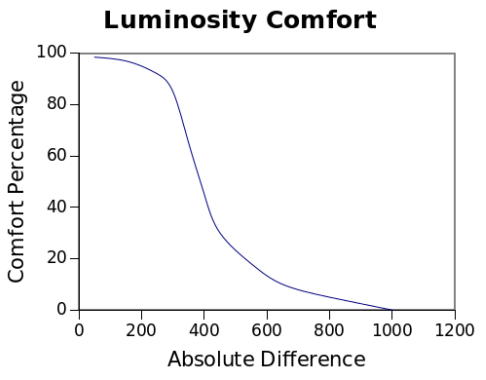

(b) Light comfort percentage depending on absolute luminosity difference.

Figure 6: Comfort percentages for the absolute difference between current and aim values.

\section{Results}

In this section, we present an evaluation of the models previously described. In particular, we firstly present results obtained when the WSN optimization model (Section 3) is applied to an office building. Once sensors and gateways are placed, we evaluate energy consumption and the comfort of occupants during a working day, applying the operational model described in Section 4

For both models, we consider the office building depicted in Fig. 1 1 and the characteristics described in Section 4

\subsection{Evaluation of the WSN deployment}

This section aims at demonstrating that the proposed planning model effectively provides energy savings while fulfilling the required constraints. The comparison is against a hypothetical complete deployment where all sensor types are placed, when possible, in each building room.

Starting from all possible locations defined in Fig. 1. the optimization model selects the positions shown in Fig. 7 . for the deployment of sensors and gateways. Green positions indicate sensors whilst orange ones are gateways. In this figure, we consider that there is no protection (i.e., the protection level is 1). Hence, the sensors are able to reach only one gateway.

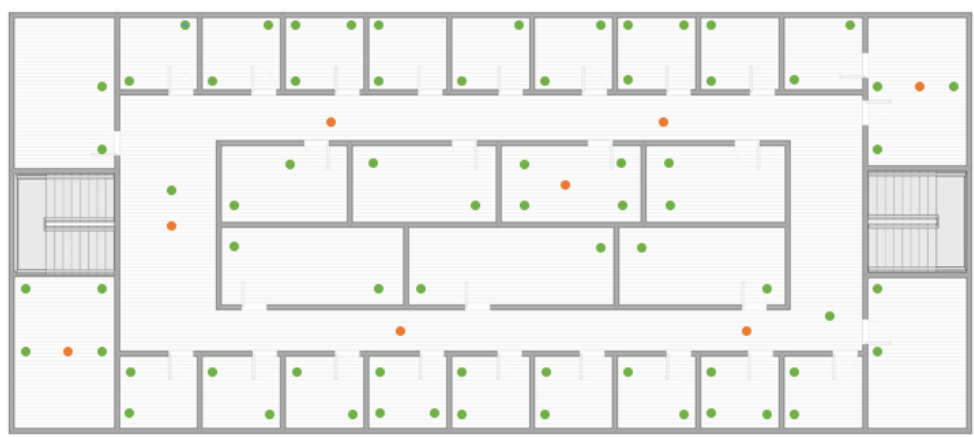

Figure 7: Sensor and gateway locations chosen by the model for the final optimized deployment.

Table 4 shows the number of sensors and gateways deployed as well as the corresponding energy consumptions for both our proposal (Optimized) and the Complete configuration, where all sensors and gateways are placed. As commented in Section 3 , for the sake of simplicity, we consider that all sensors transmit the same amount of data. As it can be seen, by optimizing the placement of sensors while still fulfilling the requirements, it is possible to reduce the number of sensors and gateways installed by $36 \%$ and $63 \%$, respectively. Regarding the energy consumption, we achieve a 37\% energy saving. 


\begin{tabular}{l|ccc} 
Network & Sensors & Gateways & Transmission Energy (nJ) \\
\hline Optimized & 69 & 8 & 54076 \\
Complete & 108 & 22 & 85384 \\
\hline
\end{tabular}

Table 4: Number of sensors and gateways installed and transmission energy consumption, according to values of Table 1 for the optimized and the complete network result.

However, it is also worth mentioning that, in the case of WSN partial failure, the Complete configuration is still able to provide full availability. To achieve a similar behaviour, we consider different levels of protection in our solution. Figure 8 shows the effect of increasing the protection level of the WSN. Remind that the protection level makes reference to the number of gateways each sensor must be able to reach in order to increase network resilience in the event of an unexpected gateway failure. For instance, if the protection level equals 1 , sensors can only reach a single gateway; at protection level 3, three gateways are reachable and only the simultaneous failure of all three would disrupt the communication.

Three cases are depicted in the figure. As in the previous results, the Optimized and Complete curves refer to our proposed model and to the case where all sensors and gateways are deployed, respectively. Note that the energy consumption for the Complete configuration is independent of the protection level. The Failure curve considers the cases in which protectionLevel - 1 gateways fail simultaneously and the sensors must, therefore, transmit to the closest available gateway. We consider the worst possible case, meaning that we remove from the network the set of protectionLevel - 1 gateways which has the highest incidence on the overall energy consumption. As it can be observed, the Optimized and Failure curves grow rapidly as the protection level increases. This is due to the increment induced by gateway failures on the distance of sensor-to-gateway transmissions. Whilst the Failure curve always stays above the Optimized curve, the difference between the two curves is not constant with the variation of the protection level. Indeed, in the worst case scenario, degradation in transmissions raises with the protection level, because of the increasing number of failing gateways. Nonetheless, it can be noticed that, with a protection level of 2 or even 3 , the additional energy consumption induced by the activation of protection is very small, especially in comparison to the energy absorbed in the Complete configuration. This entails that a substantial increment in network resilience can be provided with almost no additional cost.

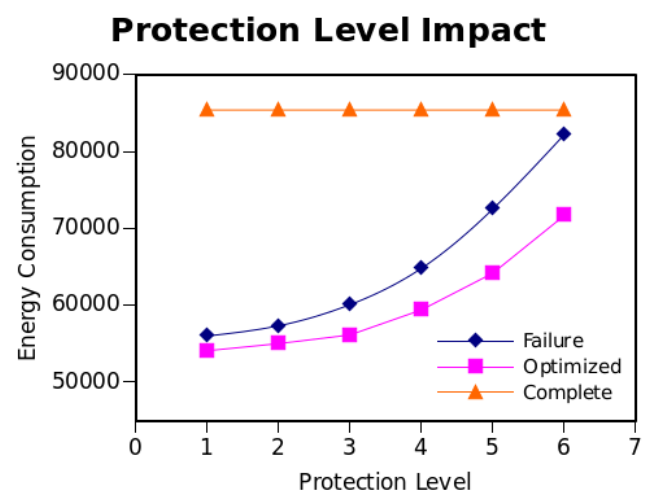

Figure 8: Network transmission energy consumption for the optimal and failure cases.

\subsection{Evaluation of the resource management}

Once the WSN is defined and deployed, the resulting layout is inserted into the operational Smart model, in order to calculate energy consumption and comfort levels under different scenarios. The comparison, in this case, is against a Base scenario which mimics as closely as possible the behaviour of a building with no automation. In particular, we consider the following Base actuations:

- HVAC systems are maintained on during the whole working period of the day, which lasts from 7am to 7pm; 
- Lights are kept on while there are someone inside the room, switched off with a 50\% probability when occupants leave the room (for meeting or lunch), and switched off at the end of the working day;

- A computer is switched on when its user enters the room, switched off with a probability of 50\% when the user leaves the room, and switched off at the end of the working day.

The specific number of occupants utilized for the simulation, grouped by profiles, is the following: 60 directors with their own offices, 150 employees using shared and private office areas, and 10 external personnel that can freely move inside the building.

The simulation considers a full day of 24 hours. The simulation is divided into steps of 10 seconds, meaning that a day is represented by 8640 steps. At each step, the simulation considers the current building status and verifies if an occupant action is expected in the near future, i.e., within the prediction threshold introduced in Section 4 For instance, if a threshold of 150 steps is considered, the BMS checks whether an action is probable in the next 25 minutes according to the occupant behavioural models. So, if an occupant is expected to be going to enter an empty room, the system acts consequently, by switching the HVAC on in advance, so that the room will be at the desired temperature when the occupant arrives.

Figure 9 shows the comparison between the Base and the Smart cases for both energy consumption and occupant comfort for different prediction thresholds.

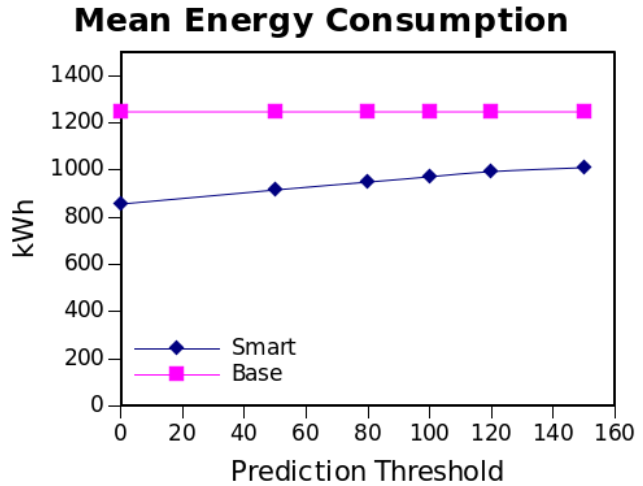

(a) Mean energy consumption in $\mathrm{kWh}$ for the different prediction thresholds (in steps) compared to the base scenario.

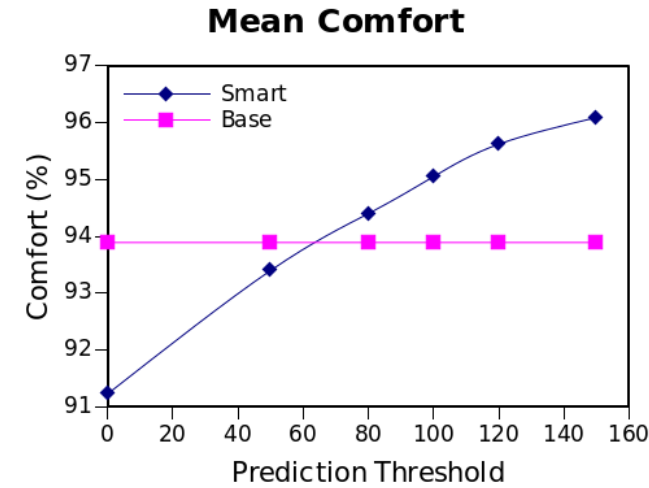

(b) Mean occupant comfort level for the different prediction thresholds (in steps) compared to the base scenario.

Figure 9: Energy and comfort comparison results between the smart and base scenario.

The final mean energy consumption presented in Fig. 9a is calculated by monitoring the status of building elements at each simulation step. As it can be observed from the Smart curve, the energy consumed by the building increases as the prediction threshold increases. This tendency lies in the fact that, having a longer prediction time and, thus, more time to react, the BMS is capable of adjusting the state of building elements beforehand in order to offer high comfort to the people yet to come (as it can be verified in Fig $9 \mathrm{~b}$ ). Moreover, it can also be seen that, even with the highest prediction threshold, energy consumption is always below the reference consumption (Base).

In the Base case, the building wastes a substantial quantity of energy by having elements, such as HVACs, unnecessarily active. For instance, it is unnecessary to have HVACs on during all working hours, since some offices may be empty during portions of the day. Moreover, manual actuation by occupants can adversely affect energy consumption if room elements are kept in a wrong state, such as leaving the lights on when a room is empty. The possibility to predict future actions and automatically actuate allows the BMS to avoid and correct such behaviours.

Figure $9 \mathrm{~b}$ presents the comparison between the mean comfort values obtained for both the Base and the Smart cases as a function of the prediction threshold. As it can be seen, in this case, the BMS is capable of delivering higher comfort than the Base scenario when the prediction threshold is high enough. When no prediction capability is granted to the system, the comfort is lower than with the Base scenario, mainly due to the latency of the HVAC 
system in reaching the desired temperature inside the building, and the inability to switch elements like lights and computers on before the arrival of the occupants. In particular, the comfort of the occupants is reduced by $2.6 \%$, but the overall energy consumed is also reduced by $31 \%$, which entails important savings. As the prediction threshold increases, the smart system is capable of adjusting room elements to create a comfortable workplace for the building occupants. Specifically, using the highest prediction threshold, the BMS is capable of increasing the overall occupant comfort by $2.2 \%$ with respect to the Base case and, at the same time, reduce the overall energy consumption by $19 \%$.

In order to better understand the efficiency of the smart building prediction system in creating appropriate conditions, we present Table 5. This table summarizes the overall number of times each room is delivered with all its inside elements in the desired state. In the Base case, a state is correct if, in an empty room, all inside elements are switched off. For the Smart case, the elements have to be switched off except iff an occupant is expected to arrive soon. When the room is occupied, the room is considered to be in a correct state iff all occupants are $95 \%$ comfortable according to Eq. 16. If at least one element is in a wrong state, the room is considered in a failure state.

\begin{tabular}{l|ccc} 
Threshold & Success & Failure & \% \\
\hline Base & 440692 & 103628 & 80.91 \\
Smart 0 & 502241 & 42079 & 92.27 \\
Smart 50 & 508145 & 36175 & 93.35 \\
Smart 80 & 510657 & 33663 & 93.82 \\
Smart 100 & 512191 & 32129 & 94.09 \\
Smart 120 & 513268 & 31052 & 94.29 \\
Smart 150 & 514164 & 30156 & 94.46 \\
\hline
\end{tabular}

Table 5: Number of success and failure room status for the base and each smart threshold simulations.

As it can be seen, the Base case offers a good environment $81 \%$ of the time, while the Smart case with no prediction is capable of delivering the correct environment $92 \%$ of the time. The main difference is because, in the case of empty rooms, the base system keeps elements on unnecessarily. The Smart system is capable of automatically turn devices off when rooms are empty, and adjust them fast enough to offer comfortable levels if an arrival is expected. As the prediction threshold increases, the success percentage also increases, getting to $94 \%$ with the maximum prediction threshold considered.

In conclusion, we have been able to determine and control the trade-off between building energy consumption and occupancy comfort. In the Smart scenarios, the comfort level can reach an average of $96 \%$ for all occupants ( $2 \%$ more than the Base case) while the energy consumption is still $19 \%$ lower than the Base case.

\section{Conclusions and Future Work}

In this paper, we have presented novel models for the planning and operational problems in smart buildings.

For the planning problem, we have proposed a MIP formulation for the optimal deployment of the building automation WSN. To this end, we have considered a model able to identify the optimal locations for different sensor types and gateways, and optimize energy consumption while fulfilling connectivity, resource, protection, and clustering coverage constraints. Obtained results show the capability of our proposal to reduce the amount of sensors and gateways needing to be deployed. For instance, we need about $37 \%$ less sensors, and $64 \%$ less gateways than with respect to the case where all sensors and gateways are placed, with an overall energy savings of $36 \%$. If protection is enforced, the number of sensors and gateways increases as well as the energy consumption. Nonetheless, with a protection level as high as 6 , the energy bill is still $18 \%$ better.

For the operational problem, we have proposed a solution that, using behavioural models, is able to efficiently configure the different building elements while maintaining, or even improving, occupant comfort. Behavioural models are used to predict the occupants' actions and act accordingly. The system is able to act proactively and configure the building in a better and faster way if a prediction threshold is adopted. For instance, we can reduce the energy consumption in a range between $20 \%$ and $32 \%$ with respect to the case with no automation. Nonetheless, the greatest savings are obtained when no prediction is used as the system acts only when the occupant actually performs an action. However, this is also associated with the lowest comfort. When a threshold is applied, the system can accurately 
predict an action and prepare the building beforehand. This improves comfort at the expense of a moderate increase in energy consumption.

As future work, we plan to enhance the prediction model. Particularly, instead of having unalterable user profile probabilities for each action, the system must be capable of understanding the occupant behaviour and evolve such probability models to reflect more closely the reality. Moreover, if such models change due to the introduction of previously unseen behaviour, the system must detect such alterations and adjust the models accordingly.

\section{References}

[1] ANSI/ASHRAE, 2004. Thermal environmental conditions for human occupancy.

[2] Barolli, A., Xhafa, F., Takizawa, M., Sept 2011. Optimization problems and resolution methods for node placement in wireless mesh networks. In: 2011 14th International Conference on Network-Based Information Systems. pp. 126-134.

[3] Belhaj, L. A., Gosselin, S., Pouyllau, H., Semet, Y., Oct 2016. Smart-sensor placement optimization under energy objectives. In: 2016 Global Information Infrastructure and Networking Symposium (GIIS). pp. 1-7.

[4] Capone, A., Cesana, M., Donno, D. D., Filippini, I., 2010. Deploying multiple interconnected gateways in heterogeneous wireless sensor networks: An optimization approach. Computer Communications 33 (10), $1151-1161$

[5] del Mar Castilla, M., Álvarez, J. D., Normey-Rico, J. E., Rodríguez, F., Berenguel, M., Nov 2013. A multivariable nonlinear mpc control strategy for thermal comfort and indoor-air quality. In: IECON 2013 - 39th Annual Conference of the IEEE Industrial Electronics Society. pp. 7908-7913.

[6] Ding, J., Xu, J., Zheng, Z., July 2009. Gateway deployment optimization in wireless mesh network: A case study in china. In: 2009 IEEE/INFORMS International Conference on Service Operations, Logistics and Informatics. pp. 300-305.

[7] Djenouri, D., Bagaa, M., Jan 2017. Energy-aware constrained relay node deployment for sustainable wireless sensor networks. IEEE Transactions on Sustainable Computing 2 (1), 30-42.

[8] Drabu, Y., Peyravi, H., April 2008. Gateway placement with qos constraints in wireless mesh networks. In: Seventh International Conference on Networking (icn 2008). pp. 46-51.

[9] Ficco, M., Palmieri, F., Castiglione, A., 2014. Hybrid indoor and outdoor location services for new generation mobile terminals. Personal and Ubiquitous Computing 18 (2), 271-285.

URL https://www.scopus.com/inward/record.uri?eid=2-s2.0-84897617754\&doi=10.1007\%2fs00779-013-0644-4\& partnerID=40\&md5=14043d76dccce8f f317b961c7c3f15ea

[10] Ghadi, Y. Y., Rasul, M., Khan, M., 2016. Design and development of advanced fuzzy logic controllers in smart buildings for institutional buildings in subtropical queensland. Renewable and Sustainable Energy Reviews 54 (Supplement C), 738 - 744.

[11] Kumar, A., Hancke, G. P., Dec 2014. An energy-efficient smart comfort sensing system based on the ieee 1451 standard for green buildings. IEEE Sensors Journal 14 (12), 4245-4252.

[12] Kumar, A., P Singh, I., K Sud, S., December 2010. An approach towards development of pmv based thermal comfort smart sensor. International Journal on Smart Sensing and Intelligent Systems 3, 621-642.

[13] National Optical Astronomy Observatory, 2015. Recommended light levels.

[14] Nguyen, T. T., Thanh, H. D., Son, L. H., Le, V. T., April 2015. Optimization for the sensor placement problem in 3d environments. In: 2015 IEEE 12th International Conference on Networking, Sensing and Control. pp. 327-333.

[15] Palmieri, F., Ficco, M., Pardi, S., Castiglione, A., 2016. A cloud-based architecture for emergency management and first responders localization in smart city environments. Computers and Electrical Engineering 56, 810-830. URL https://www.scopus.com/inward/record.uri?eid=2-s2.0-84959472416\&doi=10.1016\%2fj.compeleceng.2016.02. 012\&partnerID $=40 \& m d 5=e 45 d 1$ fed5058f $6 a 93359587 a 03 d d 244 f$

[16] Pinzon, J. A., Vergara, P. P., da Silva, L. C. P., Rider, M. J., April 2017. An milp model for optimal management of energy consumption and comfort in smart buildings. In: 2017 IEEE Power Energy Society Innovative Smart Grid Technologies Conference (ISGT). pp. 1-5.

[17] Rawi, M. I. M., Al-Anbuky, A., 2011. Development of intelligent wireless sensor networks for human comfort index measurement. Procedia Computer Science 5 (Supplement C), 232 - 239, the 2nd International Conference on Ambient Systems, Networks and Technologies (ANT2011) / The 8th International Conference on Mobile Web Information Systems (MobiWIS 2011).

[18] Reffat, R. M., Harkness, E. L., August 2001. Environmental comfort criteria: Weighting and integration. Journal of Performance of Constructed Facilities 15

[19] Roy, S., Mukherjee, N., Feb 2014. Integer linear programming formulation of optimal beacon placement problem in wsn. In: 2014 Applications and Innovations in Mobile Computing (AIMoC). pp. 111-117.

[20] Sembroiz-Ausejo, D., Ricciardi, S., Careglio, D., 2018. A novel cloud-based iot architecture for smart building automation. In: Ficco, M., Palmieri, F. (Eds.), Security and Resilience in Intelligent Data-Centric Systems and Communication Networks. Elsevier, Ch. 10, pp. $215-233$.

[21] Shaikh, P. H., Nor, N. B. M., Nallagownden, P., Elamvazuthi, I., 2016. Intelligent multi-objective optimization for building energy and comfort management. Journal of King Saud University - Engineering Sciences.

[22] Tao, M., Zuo, J., Liu, Z., Castiglione, A., Palmieri, F., 2018. Multi-layer cloud architectural model and ontology-based security service framework for iot-based smart homes. Future Generation Computer Systems 78, 1040-1051.

URL https://www.scopus.com/inward/record.uri?eid=2-s2.0-85007478607\&doi=10.1016\%2fj.future.2016.11.011\& partnerID $=40 \&$ md5 $=$ d409bcee0ea1b43d3e7837cc543704c1

[23] Vales-Alonso, J., Egea-Lopez, E., Martinez-Sala, A., Pavon-Marino, P., Bueno-Delgado, M. V., Garcia-Haro, J., 2007. Performance evaluation of mac transmission power control in wireless sensor networks. Computer Networks 51 (6), $1483-1498$.

[24] Wang, N., Fang, F., Feng, M., May 2014. Multi-objective optimal analysis of comfort and energy management for intelligent buildings. In: The 26th Chinese Control and Decision Conference (2014 CCDC). pp. 2783-2788. 